Research Article

\title{
Bounds for the Remainder in Simpson's Inequality via n-Polynomial Convex Functions of Higher Order Using Katugampola Fractional Integrals
}

\author{
Yu-Ming Chu $\mathbb{D}^{1},{ }^{1}$ Muhammad Uzair Awan $\mathbb{D}^{\circ},{ }^{2}$ Muhammad Zakria Javad, \\ and Awais Gul Khan ${ }^{2}$ \\ ${ }^{1}$ Department of Mathematics, Huzhou University, Huzhou 313000, China \\ ${ }^{2}$ Department of Mathematics, Government College University, Faisalabad, Pakistan
}

Correspondence should be addressed to Muhammad Uzair Awan; awan.uzair@gmail.com

Received 12 May 2020; Revised 24 July 2020; Accepted 24 July 2020; Published 24 August 2020

Academic Editor: Efthymios G. Tsionas

Copyright (C) $2020 \mathrm{Yu}$-Ming Chu et al. This is an open access article distributed under the Creative Commons Attribution License, which permits unrestricted use, distribution, and reproduction in any medium, provided the original work is properly cited.

\begin{abstract}
The goal of this paper is to derive some new variants of Simpson's inequality using the class of $n$-polynomial convex functions of higher order. To obtain the main results of the paper, we first derive a new generalized fractional integral identity utilizing the concepts of Katugampola fractional integrals. This new fractional integral identity will serve as an auxiliary result in the development of the main results of this paper.
\end{abstract}

\section{Introduction and Preliminaries}

The following inequality known in the literature as Simpson's inequality [1].

$$
\left|\int_{a}^{b} \Lambda(t) \mathrm{d} t-\frac{b-a}{6}\left[\Lambda(a)+4 \Lambda\left(\frac{a+b}{2}\right)+\Lambda(b)\right]\right| \leq \frac{(b-a)^{4}}{2880}\left\|\Lambda^{(4)}\right\|_{\infty},
$$

where $\Lambda:[a, b] \longrightarrow \mathbb{R}$ is a four times continuously differentiable function on $(a, b)$ and

$$
\left\|\Lambda^{(4)}\right\|_{\infty}=\sup _{t \in(a, b)}\left|\Lambda^{(4)}(t)\right|<\infty \text {. }
$$

Simpson's inequality plays a significant role in analysis [2-4]. Over the years, it has been extended and generalized in different directions using novel and innovative approaches. The survey by Dragomir et al. [5] is very informative regarding the developments of Simpson's inequality and its applications.

In recent years, the fractional calculus [6-10] is often known as noninteger calculus which has become a powerful tool in mathematics because it provides a good tool to describe physical memory. Fractional calculus has wide applications in real life through its help in solving different physical problems [11-20]. The classic definition of Riemann-Liouville fractional integrals is one of the most basic concepts in fractional calculus which is defined as:

Definition 1. Let $\Lambda \in L[a, b]$. Then Riemann-Liouville integrals $J_{a^{+}}^{\alpha} \Lambda$ and $J_{b^{-}}^{\alpha} \Lambda$ of order $\alpha>0$ with $a \geq 0$ are defined by

$$
\begin{array}{ll}
J_{a^{+}}^{\alpha} \Lambda(x)=\frac{1}{\Gamma(\alpha)} \int_{a}^{x}(x-t)^{\alpha-1} \Lambda(t) \mathrm{d} t, & x>a, \\
J_{b^{-}}^{\alpha} \Lambda(x)=\frac{1}{\Gamma(\alpha)} \int_{x}^{b}(t-x)^{\alpha-1} \Lambda(t) \mathrm{d} t, & x<b,
\end{array}
$$

where

$$
\Gamma(\alpha)=\int_{0}^{\infty} e^{-x} x^{\alpha-1} \mathrm{~d} x
$$

is a well known gamma function.

In past few decades, several successful attempts have been made in generalizing the classical concepts of fractional calculus. Erdelyi-Kober operator is a significant generalization of fractional integrals introduced and was studied by 
Arthur Erdelyi and Hermann Kober. But there is a drawback that one cannot get the Hadamard version of the derivatives and integrals from Erdelyi-Kober operators. Katugampola [21] gave a well-defined concept of fractional integrals as:

Definition 2. Let $[a, b] \subset \mathbb{R}$ be a finite interval. Then the left and right sides of Katugampola fractional integrals of order $\alpha>0$ of $\Lambda \in X_{c}^{\varrho}(a, b)$ are defined by

$$
\begin{aligned}
& { }^{\varrho} I_{a^{+}}^{\alpha} \Lambda(x)=\frac{\varrho^{1-\alpha}}{\Gamma(\alpha)} \int_{a}^{x} \frac{t^{\varrho-1}}{\left(x^{\varrho}-t^{\varrho}\right)^{1-\alpha}} \Lambda(t) \mathrm{d} t, \\
& { }^{\varrho} I_{a^{+}}^{\alpha} \Lambda(x)=\frac{\varrho^{1-\alpha}}{\Gamma(\alpha)} \int_{x}^{b} \frac{t^{\varrho-1}}{\left(t^{\varrho}-x^{\varrho}\right)^{1-\alpha}} \Lambda(t) \mathrm{d} t,
\end{aligned}
$$

if the integral exists.

If we take $\rho=1$, then we can recapture Riemann-Liouville fractional integrals from the Katugampola fractional integrals. It worth to mention here that Erdelyi-Kober operators and Katugampola fractional integrals are not equivalent to each other.

Sarikaya et al. [22] are the first authors to utilize the concepts of Riemann-Liouville fractional integrals in obtaining the fractional analogues of Hermite-Hadamard's inequality. This idea inspired several inequalities expert, and resultantly huge number of articles have been written on the fractional analogues of classical inequalities. For example, $\mathrm{Hu}$ et al. [23] obtained some new fractional analogues of integral inequalities using Katugampola fractional integrals. Nie et al. [24] obtained $k$-fractional analogues of Simpson's inequality. Peng et al. [25] also obtained some new fractional analogues of Simpson's inequality. Set [26] obtained fractional analogues of Ostrowski's inequality. Wu et al. [27] obtained fractional analogues of inequalities using $k$-th order differentiable functions. Kermausuor [28] obtained new Simpson type inequalities involving Katugampola fractional integrals essentially using the class of Breckner type $s$-convex function.

Recently, Toplu et al. [29] introduced the notion of $n$-polynomial convex functions as follows.
Definition 3. Let $n \in \mathbb{N}$. Then a nonnegative function $\Lambda: I \subset \mathbb{R} \longrightarrow \mathbb{R}$ is said to be a $n$-polynomial convex function if the inequality

$$
\Lambda\left(t x+(1-t) y \leq \frac{1}{n} \sum_{s=1}^{n}\left[1-(1-t)^{s}\right] \Lambda(x)+\frac{1}{n} \sum_{s=1}^{n}\left[1-t^{s}\right] \Lambda(y)\right.
$$

holds for every $x, y \in I$ and $t \in[0,1]$.

Many researchers have also derived several new Hermite-Hadamard's like inequalities [30-36] using the concept of $n$-polynomial convex functions. We would like to point out here that the class of $n$-polynomial convex functions generalize the class of convex functions if we take $n=1$, then we have the class of 1-polynomial convex functions which is just the classical convex functions. Also we can get other type of convexities: for example, for $n=2$, we have 2-polynomial convexity. Another point of pondering here is that every $n$-polynomial convex function is an $h$-convex function with the function $h(t)=1 / n \sum_{s=1}^{n}\left[1-(1-t)^{s}\right]$. So more generally, every nonnegative convex function is also an $n$-polynomial convex function.

The idea behind the study of this article is to extend the notion of $n$-polynomial convex functions with the introduction of higher order $n$-polynomial convex functions. We derive a new fractional integral identity using the concepts of Katugampola fractional integrals. This new identity will serve as an auxiliary result in the development of some new fractional analogues of Simpson's inequalities using the concept of $n$-polynomial convex functions of higher order.

Before we move to our main results, we would like to introduce the notion of $n$-polynomial convex functions of higher order.

Definition 4. Let $n \in \mathbb{N}$. Then a nonnegative function $\Lambda: I \subset \mathbb{R} \longrightarrow \mathbb{R}$ is said to be a higher order $n$-polynomial convex function if the inequality

$$
\Lambda\left(t x+(1-t) y \leq \frac{1}{n} \sum_{s=1}^{n}\left[1-(1-t)^{s}\right] \Lambda(x)+\frac{1}{n} \sum_{s=1}^{n}\left[1-t^{s}\right] \Lambda(y)-\mu\left(t^{\sigma}(1-t)+t(1-t)^{\sigma}\right)\|y-x\|^{\sigma}\right.
$$

holds for every $x, y \in I, \sigma>0$, and $t \in[0,1]$.

Remark 1. Note that if $\sigma=0$ in (7), then the class of $n$-polynomial convex functions of higher order reduces to the class of $n$-polynomial convex functions. If $\sigma=2$, then we have a new class of strongly $n$-polynomial convex functions. If $n=1$, then we have the class of higher-order convex functions [37]. And along with $n=1$, if we have $\sigma=2$, then the class of $n$-polynomial convex functions of higher order reduces to the class of strongly convex functions [38]. From this, it is evident that the class of $n$-polynomial convex functions of higher order is quite unifying one as it relates several other unrelated classes of convexity [39-45].

\section{Main Results}

In this section, we discuss our main results.

2.1. A Key Lemma. We now derive the main auxiliary result of the paper. 
Journal of Mathematics

3

Lemma 1. Let $\alpha, \varrho>0$, and let $\Lambda:\left[a^{\varrho}, b^{\varrho}\right] \longrightarrow \mathbb{R}$ be a differentiable function on $\left(a^{\varrho}, b^{\varrho}\right)$, with $0 \leq \alpha<b$ such that $\Lambda^{\prime} \in L_{1}\left(\left[a^{\varrho}, b^{\varrho}\right]\right)$, then

$$
\begin{aligned}
& \frac{1}{(i+1)(i+2)}\left[\Lambda\left(a^{\varrho}\right)+(i+1)\left(\Lambda\left(\frac{i a^{\varrho}+b^{\varrho}}{i+1}\right)+\Lambda\left(\frac{a^{\varrho}+i b^{\varrho}}{i+1}\right)\right)+\Lambda\left(b^{\varrho}\right)\right] \\
& -\frac{(i+1)^{\alpha-1} \varrho^{\alpha} \Gamma(\alpha+1)}{\left(b^{\varrho}-a^{\varrho}\right)^{\alpha}}\left[{ }^{\varrho} I_{a^{+}}^{\alpha} \Lambda\left(\frac{i a^{\varrho}+b^{\varrho}}{i+1}\right)+{ }^{\varrho} I_{b^{-}}^{\alpha} \Lambda\left(\frac{a^{\varrho}+i b^{\varrho}}{i+1}\right)\right] \\
& =\frac{\varrho\left(b^{\varrho}-a^{\varrho}\right)}{i+1}\left[\int_{0}^{1}\left(\frac{1}{i+2}-\frac{t^{\alpha \varrho}}{i+1}\right) t^{\varrho-1} \Lambda^{\prime}\left(\frac{i+t^{\varrho}}{i+1} a^{\varrho}+\frac{1-t^{\varrho}}{i+1} b^{\varrho}\right) \mathrm{d} t-\int_{0}^{1}\left(\frac{1}{i+2}-\frac{t^{\alpha \varrho}}{i+1}\right) t^{\varrho-1} \Lambda^{\prime}\left(\frac{1-t^{\varrho}}{i+1} a^{\varrho}+\frac{i+t^{\varrho}}{i+1} b^{\varrho}\right) \mathrm{d} t\right] .
\end{aligned}
$$

Proof. Consider

$$
\begin{aligned}
& I=\frac{\varrho\left(b^{\varrho}-a^{\varrho}\right)}{i+1}\left[\int_{0}^{1}\left(\frac{1}{i+2}-\frac{t^{\alpha}}{i+1}\right) t^{\varrho-1} \Lambda^{\prime}\left(\frac{i+t^{\varrho}}{i+1} a^{\varrho}+\frac{1-t^{\varrho}}{i+1} b^{\varrho}\right) \mathrm{d} t-\int_{0}^{1}\left(\frac{1}{i+2}-\frac{t^{\alpha}}{i+1} t^{\varrho^{-1}} \Lambda^{\prime}\left(\frac{1-t^{\varrho}}{i+1} a^{\varrho}+\frac{i+t^{\varrho}}{i+1} b^{\varrho}\right) \mathrm{d} t\right)\right] \\
& I=\frac{\varrho\left(b^{\varrho}-a^{\varrho}\right)}{i+1}\left[I_{1}-I_{2}\right] .
\end{aligned}
$$

Integrating by parts $I_{1}$, we have

$$
\begin{aligned}
I_{1} & =\int_{0}^{1}\left(\frac{1}{i+2}-\frac{t^{\alpha \varrho}}{i+1}\right) t^{\varrho^{-1}} \Lambda^{\prime}\left(\frac{i+t^{\varrho}}{i+1} a^{\varrho}+\frac{1-t^{\varrho}}{i+1} b^{\varrho}\right) \mathrm{d} t \\
& =-\left.\frac{i+1}{\varrho\left(b^{\varrho}-a^{\varrho}\right)}\left(\frac{1}{i+2}-\frac{t^{\alpha \varrho}}{i+1}\right) \Lambda\left(\frac{i+t^{\varrho}}{i+1} a^{\varrho}+\frac{1-t^{\varrho}}{i+1} b^{\varrho}\right)\right|_{0} ^{1}-\frac{\alpha}{b^{\varrho}-a^{\varrho}} \int_{0}^{1} t^{\alpha \varrho-1} \Lambda\left(\frac{i+t^{\varrho}}{i+1} a^{\varrho}+\frac{1-t^{\varrho}}{i+1} b^{\varrho}\right) \mathrm{d} t, \\
& =\frac{\Lambda\left(a^{\varrho}\right)}{\varrho\left(b^{\varrho}-a^{\varrho}\right)(i+2)}+\frac{i+1}{\varrho(i+2)\left(b^{\varrho}-a^{\varrho}\right)} \Lambda\left(\frac{i a^{\varrho}+b^{\varrho}}{i+1}\right)-\frac{(i+1)^{\alpha} \varrho^{\alpha-1} \Gamma(\alpha+1)^{\varrho}}{\left(b^{\varrho}-a^{\varrho}\right)^{\alpha+1}} I_{a^{+}}^{\alpha} \Lambda\left(\frac{i a^{\varrho}+b^{\varrho}}{i+1}\right) .
\end{aligned}
$$

Similarly,

$$
\begin{aligned}
I_{2} & =\int_{0}^{1}\left(\frac{1}{i+2}-\frac{t^{\alpha}}{i+1}\right) t^{\varrho-1} \Lambda^{\prime}\left(\frac{1-t^{\varrho}}{i+1} a^{\varrho}+\frac{i+t^{\varrho}}{i+1} b^{\varrho}\right) \mathrm{d} t \\
& =-\frac{\Lambda\left(b^{\varrho}\right)}{\varrho\left(b^{\varrho}-a^{\varrho}\right)(i+2)}-\frac{i+1}{\varrho(i+2)\left(b^{\varrho}-a^{\varrho}\right)} \Lambda\left(\frac{a^{\varrho}+i b^{\varrho}}{i+1}\right)+\frac{(i+1)^{\alpha} \varrho^{\alpha-1} \Gamma(\alpha+1)^{\varrho}}{\left(b^{\varrho}-a^{\varrho}\right)^{\alpha+1}} I_{b^{\alpha}}^{\alpha} \Lambda\left(\frac{a^{\varrho}+i b^{\varrho}}{i+1}\right) .
\end{aligned}
$$


By substituting the values of $I_{1}$ and $I_{2}$ in $I$, we get the required result.

This completes the proof.
Theorem 1. Let $\alpha, \varrho>0$ and let $\Lambda:\left[a^{\varrho}, b^{\varrho}\right] \longrightarrow \mathbb{R}$ be $a$ differentiable function on $\left(a^{\varrho}, b^{\varrho}\right)$, with $0 \leq \alpha<b$ such that $\Lambda^{\prime} \in L_{1}\left(\left[a^{\varrho}, b^{\varrho}\right]\right)$. If $\left|\Lambda^{\prime}\right|$ is higher-order n-polynomial convex function of order $\sigma>0$, then

2.2. Results and Discussions. We now derive the main results using Lemma 1.

$$
\begin{aligned}
& \mid \frac{1}{(i+1)(i+2)}\left[\Lambda\left(a^{\varrho}\right)+\Lambda\left(b^{\varrho}\right)+(i+1)\left(\Lambda\left(\frac{i a^{\varrho}+b^{\varrho}}{i+1}\right)+\Lambda\left(\frac{a^{\varrho}+i b^{\varrho}}{i+1}\right)\right)\right]-\frac{(i+1)^{\alpha-1} \varrho^{\alpha} \Gamma(\alpha+1)}{\left(b^{\varrho}-a^{\varrho}\right)^{\alpha}}\left[\varrho I_{a^{+}}^{\alpha} \Lambda\left(\frac{i a^{\varrho}+b^{\varrho}}{i+1}\right)\right. \\
& \left.\quad+{ }^{\varrho} I_{b^{-}}^{\alpha} \Lambda\left(\frac{a^{\varrho}+i b^{\varrho}}{i+1}\right)\right] \\
& \quad \leq \frac{\left(b^{\varrho}-a^{\varrho}\right)}{(i+1)(i+2)}\left[\left(\frac{1}{n} \sum_{s=1}^{n} \frac{(i+1)^{s}(2 s-i+1)+i^{s+1}-1}{(i+1)^{s}(s+1)}\left(\left|\Lambda^{\prime}\left(a^{\varrho}\right)\right|+\left|\Lambda^{\prime}\left(b^{\varrho}\right)\right|\right)\right)-\frac{4 \mu}{(\sigma+1)(\sigma+2)}\left\|b^{\varrho}-a^{\varrho}\right\|^{\sigma}\right] .
\end{aligned}
$$

Proof. By using Lemma 1 and because $\left|\Lambda^{\prime}\right|$ is higher-order $n$-polynomial convex function of order $\sigma>0$, we have

$$
\begin{aligned}
& \mid \frac{1}{(i+1)(i+2)}\left[\Lambda\left(a^{\varrho}\right)+\Lambda\left(b^{\varrho}\right)+(i+1)\left(\Lambda\left(\frac{i a^{\varrho}+b^{\varrho}}{i+1}\right)+\Lambda\left(\frac{a^{\varrho}+i b^{\varrho}}{i+1}\right)\right)\right]-\frac{(i+1)^{\alpha-1} \varrho^{\alpha} \Gamma(\alpha+1)}{\left(b^{\varrho}-a^{\varrho}\right)^{\alpha}}\left[{ }^{\varrho} I_{a^{+}}^{\alpha} \Lambda\left(\frac{i a^{\varrho}+b^{\varrho}}{i+1}\right)\right. \\
& \left.\quad+{ }^{\varrho} I_{b^{-}}^{\alpha} \Lambda\left(\frac{a^{\varrho}+i b^{\varrho}}{i+1}\right)\right] \mid \\
& \quad \leq \frac{\varrho\left(b^{\varrho}-a^{\varrho}\right)}{i+1}\left[\int_{0}^{1}\left|\frac{1}{i+2}-\frac{t^{\alpha}}{i+1}\right| t^{\varrho^{-1}}\left|\Lambda^{\prime}\left(\frac{i+t^{\varrho}}{i+1} a^{\varrho}+\frac{1-t^{\varrho}}{i+1} b^{\varrho}\right)\right| \mathrm{d} t+\int_{0}^{1}\left|\frac{1}{i+2}-\frac{t^{\alpha}}{i+1}\right| t^{\varrho^{-1}}\left|\Lambda^{\prime}\left(\frac{1-t^{\varrho}}{i+1} a^{\varrho}+\frac{i+t^{\varrho}}{i+1} b^{\varrho}\right)\right| \mathrm{d} t\right] \\
& \quad=\frac{\varrho\left(b^{\varrho}-a^{\varrho}\right)}{i+1}\left[I_{1}+I_{2}\right] .
\end{aligned}
$$

Note that

$$
\begin{aligned}
I_{1}= & \int_{0}^{1}\left|\frac{1}{i+2}-\frac{t^{\alpha}}{i+1}\right| t^{\varrho-1}\left|\Lambda^{\prime}\left(\frac{i+t^{\varrho}}{i+1} a^{\varrho}+\frac{1-t^{\varrho}}{i+1} b^{\varrho}\right)\right| \mathrm{d} t \\
= & \frac{1}{\varrho} \int_{0}^{1}\left|\frac{1}{i+2}-\frac{x^{\alpha}}{i+1}\right|\left|\Lambda^{\prime}\left(\frac{i+x}{i+1} a^{\varrho}+\frac{1-x}{i+1} b^{\varrho}\right)\right| \mathrm{d} x \\
\leq & \frac{1}{\varrho}\left[\left(\int_{0}^{1}\left|\frac{1}{i+2}-\frac{x^{\alpha}}{i+1}\right| \mathrm{d} x\right)\left(\int_{0}^{1}\left|\Lambda^{\prime}\left(\frac{i+x}{i+1} a^{\varrho}+\frac{1-x}{i+1} b^{\varrho}\right)\right| \mathrm{d} x\right)\right] \\
\leq & \frac{1}{\varrho}\left(\int_{0}^{1}\left|\frac{1}{i+2}-\frac{x^{\alpha}}{i+1}\right| \mathrm{d} x\right)\left(\int _ { 0 } ^ { 1 } \left(\frac{1}{n} \sum_{s=1}^{n}\left(1-\left(1-\left(\frac{i+x}{i+1}\right)\right)^{s}\right)\left|\Lambda^{\prime}\left(a^{\varrho}\right)\right|\right.\right. \\
& \left.\left.+\frac{1}{n} \sum_{s=1}^{n}\left(1-\left(\frac{i+x}{i+1}\right)^{s}\right)\left|\Lambda^{\prime}\left(b^{\varrho}\right)\right|-\mu\left(x^{\sigma}(1-x)+x(1-x)^{\sigma}\right)\left\|b^{\varrho}-a^{\varrho}\right\|^{\sigma} \mathrm{d} x\right)\right) \\
= & \frac{1}{\varrho(i+2)}\left[\left(\frac{1}{n} \sum_{s=1}^{n} \frac{(i+1)^{s}(1+s)-1}{(i+1)^{s}(1+s)}\left|\Lambda^{\prime}\left(a^{\varrho}\right)\right|+\frac{1}{n} \sum_{s=1}^{n} \frac{(i+1)^{s}(s-i)+i^{s+1}}{(i+1)^{s}(1+s)}\left|\Lambda^{\prime}\left(b^{\varrho}\right)\right|-\frac{2 \mu}{(\sigma+1)(\sigma+2)}\left\|b^{\varrho}-a^{\varrho}\right\|^{\sigma}\right)^{1 / q}\right] .
\end{aligned}
$$


Similarly,

$$
\begin{aligned}
I_{2}= & \int_{0}^{1}\left|\frac{1}{i+2}-\frac{t^{\alpha}}{i+1}\right| t^{\varrho-1}\left|\Lambda^{\prime}\left(\frac{1-t^{\varrho}}{i+1} a^{\varrho}+\frac{i+t^{\varrho}}{i+1} b^{\varrho}\right)\right| \mathrm{d} t \\
= & \frac{1}{\varrho} \int_{0}^{1}\left|\frac{1}{i+2}-\frac{x^{\alpha}}{i+1}\right| \Lambda^{\prime}\left(\frac{1-x}{i+1} a^{\varrho}+\frac{i+x}{i+1} b^{\varrho}\right) \mid \mathrm{d} x \\
\leq & \frac{1}{\varrho}\left[\left(\int_{0}^{1}\left|\frac{1}{i+2}-\frac{x^{\alpha}}{i+1}\right| \mathrm{d} x\right)\left(\int_{0}^{1}\left|\Lambda^{\prime}\left(\frac{1-x}{i+1} a^{\varrho}+\frac{i+x}{i+1} b^{\varrho}\right)\right| \mathrm{d} x\right)\right] \\
\leq & \frac{1}{\varrho}\left[( \int _ { 0 } ^ { 1 } | \frac { 1 } { i + 2 } - \frac { x ^ { \alpha } } { i + 1 } | \mathrm { d } x ) \left(\int _ { 0 } ^ { 1 } \left(\frac{1}{n} \sum_{s=1}^{n}\left(1-\left(1-\left(\frac{1-x}{i+1}\right)\right)^{s}\right)\left|\Lambda^{\prime}\left(a^{\varrho}\right)\right|\right.\right.\right. \\
& \left.\left.\left.+\frac{1}{n} \sum_{s=1}^{n}\left(1-\left(\frac{1-x}{i+1}\right)^{s}\right)\left|\Lambda^{\prime}\left(b^{\varrho}\right)\right|-\mu\left(x^{\sigma}(1-x)+x(1-x)^{\sigma}\right)\left\|b^{\varrho}-a^{\varrho}\right\|^{\sigma} \mathrm{d} x\right)\right)\right] \\
= & \frac{1}{\varrho(i+2)}\left[\left(\frac{1}{n} \sum_{s=1}^{n} \frac{(i+1)^{s}(s-i)+i^{s+1}}{(i+1)^{s}(1+s)}\left|\Lambda^{\prime}\left(a^{\varrho}\right)\right|+\frac{1}{n} \sum_{s=1}^{n} \frac{(i+1)^{s}(1+s)-1}{(i+1)^{s}(1+s)}\left|\Lambda^{\prime}\left(b^{\varrho}\right)\right|-\frac{2 \mu}{(\sigma+1)(\sigma+2)}\left\|b^{\varrho}-a^{\varrho}\right\|^{\sigma}\right)\right],
\end{aligned}
$$

where we have used the fact that $\left|1 / i+2-x^{\alpha} / i+1\right| \leq 1 / i+2$ for all $x \in[0,1]$. Using $I_{1}$ and $I_{2}$ in (13), we get the required result.
Theorem 2. Let $\alpha, \varrho>0$, and let $\Lambda:\left[a^{\varrho}, b^{\varrho}\right] \longrightarrow \mathbb{R}$ be $a$ differentiable function on $\left(a^{\varrho}, b^{\varrho}\right)$, with $0 \leq \alpha<b$ such that $\Lambda^{\prime} \in L_{1}\left(\left[a^{\varrho}, b^{\varrho}\right]\right)$. If $\left|\Lambda^{\prime}\right|^{q}$ is higher-order $n$-polynomial convex function of order $\sigma>0$, where $r^{-1}+q^{-1}=1$ and $q>1$, then

$$
\begin{aligned}
& \mid \frac{1}{(i+1)(i+2)}\left[\Lambda\left(a^{\varrho}\right)+\Lambda\left(b^{\varrho}\right)+(i+1)\left(\Lambda\left(\frac{i a^{\varrho}+b^{\varrho}}{i+1}\right)+\Lambda\left(\frac{a^{\varrho}+i b^{\varrho}}{i+1}\right)\right)\right] \\
& \quad-\frac{(i+1)^{\alpha-1} \varrho^{\alpha} \Gamma(\alpha+1)}{\left(b^{\varrho}-a^{\varrho}\right)^{\alpha}}\left[{ }^{\varrho} I_{a^{+}}^{\alpha} \Lambda\left(\frac{i a^{\varrho}+b^{\varrho}}{i+1}\right)+{ }^{\varrho} I_{b^{-}}^{\alpha} \Lambda\left(\frac{a^{\varrho}+i b^{\varrho}}{i+1}\right)\right] \mid \\
& \quad \leq \frac{\left(b^{\varrho}-a^{\varrho}\right)}{(i+1)(i+2)}\left[\left(\frac{1}{n} \sum_{s=1}^{n} \frac{(i+1)^{s}(1+s)-1}{(i+1)^{s}(1+s)}\left|\Lambda^{\prime}\left(a^{\varrho}\right)\right|^{q}+\frac{1}{n} \sum_{s=1}^{n} \frac{(i+1)^{s}(s-i)+i^{s+1}}{(i+1)^{s}(1+s)}\left|\Lambda^{\prime}\left(b^{\varrho}\right)\right|^{q}-\frac{2 \mu}{(\sigma+1)(\sigma+2)^{2}}\left\|b^{\varrho}-a^{\varrho}\right\|^{\sigma}\right)\right. \\
& \left.\quad+\left(\frac{1}{n} \sum_{s=1}^{n} \frac{(i+1)^{s}(s-i)+i^{s+1}}{(i+1)^{s}(1+s)}\left|\Lambda^{\prime}\left(a^{\varrho}\right)\right|^{q}+\frac{1}{n} \sum_{s=1}^{n} \frac{(i+1)^{s}(1+s)-1}{(i+1)^{s}(1+s)}\left|\Lambda^{\prime}\left(b^{\varrho}\right)\right|^{q}-\frac{2 \mu}{(\sigma+1)(\sigma+2)^{2}}\left\|b^{\varrho}-a^{\varrho}\right\|^{\sigma}\right)^{1 / q}\right] .
\end{aligned}
$$

Proof. Using Lemma 1, Holder's inequality and because $|\Lambda,|^{q}$ is a higher-order $n$-polynomial convex function of order $\sigma>0$, we have

$$
\begin{aligned}
& \mid \frac{1}{(i+1)(i+2)}\left[\Lambda\left(a^{\varrho}\right)+\Lambda\left(b^{\varrho}\right)+(i+1)\left(\Lambda\left(\frac{i a^{\varrho}+b^{\varrho}}{i+1}\right)+\Lambda\left(\frac{a^{\varrho}+i b^{\varrho}}{i+1}\right)\right)\right] \\
& \quad-\frac{(i+1)^{\alpha-1} \varrho^{\alpha} \Gamma(\alpha+1)}{\left(b^{\varrho}-a^{\varrho}\right)^{\alpha}}\left[{ }^{\varrho} I_{a^{+}}^{\alpha} \Lambda\left(\frac{i a^{\varrho}+b^{\varrho}}{i+1}\right)+{ }^{\varrho} I_{b^{-}}^{\alpha} \Lambda\left(\frac{a^{\varrho}+i b^{\varrho}}{i+1}\right)\right] \mid \\
& \quad \leq \frac{\varrho\left(b^{\varrho}-a^{\varrho}\right)}{i+1}\left[\int_{0}^{1}\left|\frac{1}{i+2}-\frac{t^{\alpha \varrho}}{i+1}\right| t^{\varrho-1}\left|\Lambda^{\prime}\left(\frac{i+t^{\varrho}}{i+1} a^{\varrho}+\frac{1-t^{\varrho}}{i+1} b^{\varrho}\right)\right| \mathrm{d} t+\int_{0}^{1}\left|\frac{1}{i+2}-\frac{t^{\alpha \varrho}}{i+1}\right| t^{\varrho-1}\left|\Lambda^{\prime}\left(\frac{1-t^{\varrho}}{i+1} a^{\varrho}+\frac{i+t^{\varrho}}{i+1} b^{\varrho}\right)\right| \mathrm{d} t\right] \\
& =\frac{\varrho\left(b^{\varrho}-a^{\varrho}\right)}{i+1}\left[I_{3}+I_{4}\right] .
\end{aligned}
$$

Note that 


$$
\begin{aligned}
I_{3}= & \int_{0}^{1}\left|\frac{1}{i+2}-\frac{t^{\alpha}}{i+1}\right| t^{\varrho^{-1}}\left|\Lambda^{\prime}\left(\frac{i+t^{\varrho}}{i+1} a^{\varrho}+\frac{1-t^{\varrho}}{i+1} b^{\varrho}\right)\right| \mathrm{d} t \\
= & \frac{1}{\varrho} \int_{0}^{1}\left|\frac{1}{i+2}-\frac{x^{\alpha}}{i+1}\right|\left|\Lambda^{\prime}\left(\frac{i+x}{i+1} a^{\varrho}+\frac{1-x}{i+1} b^{\varrho}\right)\right| \mathrm{d} x \\
\leq & \frac{1}{\varrho}\left[\left(\int_{0}^{1}\left|\frac{1}{i+2}-\frac{x^{\alpha}}{i+1}\right|^{r} \mathrm{~d} x\right)^{1 / r}\left(\int_{0}^{1}\left|\Lambda^{\prime}\left(\frac{i+x}{i+1} a^{\varrho}+\frac{1-x}{i+1} b^{\varrho}\right)\right|^{q} \mathrm{~d} x\right)^{1 / q}\right] \\
\leq & \frac{1}{\varrho}\left[\left[( \int _ { 0 } ^ { 1 } | \frac { 1 } { i + 2 } - \frac { x ^ { \alpha } } { i + 1 } | ^ { r } \mathrm { d } x ) ^ { 1 / r } \left(\left(\int _ { 0 } ^ { 1 } \left(\frac{1}{n} \sum_{s=1}^{n}\left(1-\left(1-\left(\frac{i+x}{i+1}\right)\right)^{s}\right)\left|\Lambda^{\prime}\left(a^{\varrho}\right)\right|^{q}+\frac{1}{n} \sum_{s=1}^{n}\left(1-\left(\frac{i+x}{i+1}\right)^{s}\right)\left|\Lambda^{\prime}\left(b^{\varrho}\right)\right|^{q}\right.\right.\right.\right.\right. \\
& \left.\left.\left.\left.-\mu\left(x^{\sigma}(1-x)+x(1-x)^{\sigma}\right)\left\|b^{\varrho}-a^{\varrho}\right\|^{\sigma} \mathrm{d} x\right)^{1 / q}\right)\right]\right] \\
= & \frac{1}{\varrho(i+2)}\left[\left(\frac{1}{n} \sum_{s=1}^{n} \frac{(i+1)^{s}(1+s)-1}{(i+1)^{s}(1+s)}\left|\Lambda^{\prime}\left(a^{\varrho}\right)\right|^{q}+\frac{1}{n} \sum_{s=1}^{n} \frac{(i+1)^{s}(s-i)+i^{s+1}}{(i+1)^{s}(1+s)}\left|\Lambda^{\prime}\left(b^{\varrho}\right)\right|^{q}-\frac{2 \mu}{(\sigma+1)(\sigma+2)^{2}}\left\|b^{\varrho}-a^{\varrho}\right\|^{\sigma}\right)^{1 / q}\right] .
\end{aligned}
$$

Similarly,

$$
\begin{aligned}
I_{4} & =\int_{0}^{1}\left|\frac{1}{i+2}-\frac{t^{\alpha \varrho}}{i+1}\right| t^{\varrho^{-1}}\left|\Lambda^{\prime}\left(\frac{1-t^{\varrho}}{i+1} a^{\varrho}+\frac{i+t^{\varrho}}{i+1} b^{\varrho}\right)\right| \mathrm{d} t \\
& =\frac{1}{\varrho} \int_{0}^{1}\left|\frac{1}{i+2}-\frac{x^{\alpha}}{i+1}\right| \Lambda^{\prime}\left(\frac{1-x}{i+1} a^{\varrho}+\frac{i+x}{i+1} b^{\varrho}\right) \mid \mathrm{d} x \\
& \leq \frac{1}{\varrho}\left[\left(\int_{0}^{1}\left|\frac{1}{i+2}-\frac{x^{\alpha}}{i+1}\right|^{r} \mathrm{~d} x\right)^{1 / r}\left(\int_{0}^{1}\left|\Lambda^{\prime}\left(\frac{1-x}{i+1} a^{\varrho}+\frac{i+x}{i+1} b^{\varrho}\right)\right|^{q} \mathrm{~d} x\right)^{1 / q}\right] \\
& \leq \frac{1}{\varrho}\left[\left(\int_{0}^{1}\left|\frac{1}{i+2}-\frac{x^{\alpha}}{i+1}\right|^{r} \mathrm{~d} x\right)^{1 / r}\left(\int_{0}^{1}\left(\frac{1}{n} \sum_{s=1}^{n}\left(1-\left(1-\left(\frac{1-x}{i+1}\right)\right)^{s}\right)\left|\Lambda^{\prime}\left(a^{\varrho}\right)\right|^{q}+\frac{1}{n} \sum_{s=1}^{n}\left(1-\left(\frac{1-x}{i+1}\right)^{s}\right)\left|\Lambda^{\prime}\left(b^{\varrho}\right)\right|^{q}-\mu\left(x^{\sigma}(1-x)+x(1-x)^{\sigma}\right)\left\|b^{\varrho}-a^{\varrho}\right\|^{\sigma} \mathrm{d} x\right)\right)^{1 / q}\right] \\
& =\frac{1}{\varrho(i+2)}\left[\left(\frac{1}{n} \sum_{s=1}^{n} \frac{(i+1)^{s}(s-i)+i^{s+1}}{(i+1)^{s}(1+s)}\left|\Lambda^{\prime}\left(a^{\varrho}\right)\right|^{q}+\frac{1}{n} \sum_{s=1}^{n} \frac{(i+1)^{s}(1+s)-1}{(i+1)^{s}(1+s)}\left|\Lambda^{\prime}\left(b^{\varrho}\right)\right|^{q}-\frac{2 \mu}{(\sigma+1)(\sigma+2)}\left\|b^{\varrho}-a^{\varrho}\right\|^{\sigma}\right)^{1 / q}\right],
\end{aligned}
$$

where $\left|1 / i+2-x^{\alpha} / i+1\right| \leq 1 / i+2$ for all $x \in[0,1]$. Using $I_{3}$ and $I_{4}$ in (17), we get the required result.

$$
\begin{aligned}
& \mid \frac{1}{(i+1)(i+2)}\left[\Lambda\left(a^{\varrho}\right)+\Lambda\left(b^{\varrho}\right)+(i+1)\left(\Lambda\left(\frac{i a^{\varrho}+b^{\varrho}}{i+1}\right)+\Lambda\left(\frac{a^{\varrho}+i b^{\varrho}}{i+1}\right)\right)\right] \\
& \quad-\frac{(i+1)^{\alpha-1} \varrho^{\alpha} \Gamma(\alpha+1)}{\left(b^{\varrho}-a^{\varrho}\right)^{\alpha}}\left[{ }^{\varrho} I_{a^{+}}^{\alpha} \Lambda\left(\frac{i a^{\varrho}+b^{\varrho}}{i+1}\right)+{ }^{\varrho} I_{b^{-}}^{\alpha} \Lambda\left(\frac{a^{\varrho}+i b^{\varrho}}{i+1}\right)\right] \mid \\
& \leq \frac{\left(b^{\varrho}-a^{\varrho}\right)}{(i+1)(i+2)^{1 / r}}\left[\begin{array}{l}
\left.\left(\frac{1}{i(i+2)} \sum_{s=1}^{n} \frac{(i+1)^{s}(1+s)-1}{(i+1)^{s}(1+s)}\left|\Lambda^{\prime}\left(a^{\varrho}\right)\right|^{q}+\frac{1}{n(i+2)} \sum_{s=1}^{n} \frac{(i+1)^{s}(s-i)+i^{s+1}}{(i+1)^{s}(1+s)}\left|\Lambda^{\prime}\left(b^{\varrho}\right)\right|^{q}-\frac{2 \mu}{(i+2)(\sigma+1)(\sigma+2)}\left\|b^{\varrho}-a^{\varrho}\right\|^{\sigma}\right)^{1 / q}\right] \\
\left.+\left(\frac{1}{n(i+2)} \sum_{s=1}^{n} \frac{(i+1)^{s}(s-i)+i^{s+1}}{(i+1)^{s}(1+s)}\left|\Lambda^{\prime}\left(a^{\varrho}\right)\right|^{q}+\frac{1}{n(i+2)} \sum_{s=1}^{n} \frac{(i+1)^{s}(1+s)-1}{(i+1)^{s}(1+s)}\left|\Lambda^{\prime}\left(b^{\varrho}\right)\right|^{q}-\frac{2 \mu}{(i+2)(\sigma+1)(\sigma+2)^{\prime}}\left\|b^{\varrho}-a^{\varrho}\right\|^{\sigma}\right)^{1 / q}\right] .
\end{array}\right.
\end{aligned}
$$

Theorem 3. Let $\alpha, \varrho>0$, and let $\Lambda:\left[a^{\varrho}, b^{\varrho}\right] \longrightarrow \mathbb{R}$ be $a$ differentiable function on $\left(a^{\varrho}, b^{\varrho}\right)$, with $0 \leq \alpha<b$ such that $\Lambda^{\prime} \in L_{1}\left(\left[a^{\varrho}, b^{\varrho}\right]\right)$. If $|\Lambda|^{q}$ is a higher-order n-polynomial convex function of order $\sigma>0$ where $q>1$, then 
Journal of Mathematics

7

Proof. Using Lemma 1, Power mean inequality, and because $|\Lambda /|^{q}$ is a higher-order $n$-polynomial convex function of order $\sigma>0$, we have

$$
\begin{aligned}
& \mid \frac{1}{(i+1)(i+2)}\left[\Lambda\left(a^{\varrho}\right)+\Lambda\left(b^{\varrho}\right)+(i+1)\left(\Lambda\left(\frac{i a^{\varrho}+b^{\varrho}}{i+1}\right)+\Lambda\left(\frac{a^{\varrho}+i b^{\varrho}}{i+1}\right)\right)\right] \\
& \quad-\frac{(i+1)^{\alpha-1} \varrho^{\alpha} \Gamma(\alpha+1)}{\left(b^{\varrho}-a^{\varrho}\right)^{\alpha}}\left[{ }^{\varrho} I_{a^{+}}^{\alpha} \Lambda\left(\frac{i a^{\varrho}+b^{\varrho}}{i+1}\right)+{ }^{\varrho} I_{b^{-}}^{\alpha} \Lambda\left(\frac{a^{\varrho}+i b^{\varrho}}{i+1}\right)\right] \mid \\
& \leq \frac{\varrho\left(b^{\varrho}-a^{\varrho}\right)}{i+1}\left[\int_{0}^{1}\left|\frac{1}{i+2}-\frac{t^{\alpha \varrho}}{i+1}\right| t^{\varrho^{-1}}\left|\Lambda^{\prime}\left(\frac{i+t^{\varrho}}{i+1} a^{\varrho}+\frac{1-t^{\varrho}}{i+1} b^{\varrho}\right)\right| \mathrm{d} t+\int_{0}^{1}\left|\frac{1}{i+2}-\frac{t^{\alpha \varrho}}{i+1}\right| t^{\varrho-1}\left|\Lambda^{\prime}\left(\frac{1-t^{\varrho}}{i+1} a^{\varrho}+\frac{i+t^{\varrho}}{i+1} b^{\varrho}\right)\right| \mathrm{d} t\right] \\
& =\frac{\varrho\left(b^{\varrho}-a^{\varrho}\right)}{i+1}\left[I_{5}+I_{6}\right] .
\end{aligned}
$$

Note that

$$
\begin{aligned}
I_{5}= & \int_{0}^{1}\left|\frac{1}{i+2}-\frac{t^{\alpha}}{i+1}\right| t^{\varrho-1}\left|\Lambda^{\prime}\left(\frac{i+t^{\varrho}}{i+1} a^{\varrho}+\frac{1-t^{\varrho}}{i+1} b^{\varrho}\right)\right| \mathrm{d} t \\
= & \frac{1}{\varrho} \int_{0}^{1}\left|\frac{1}{i+2}-\frac{x^{\alpha}}{i+1}\right|\left|\Lambda^{\prime}\left(\frac{i+x}{i+1} a^{\varrho}+\frac{1-x}{i+1} b^{\varrho}\right)\right| \mathrm{d} x \\
\leq & \frac{1}{\varrho}\left[\left(\int_{0}^{1}\left|\frac{1}{i+2}-\frac{x^{\alpha}}{i+1}\right| \mathrm{d} x\right)^{1 / r}\left(\int_{0}^{1}\left|\frac{1}{i+2}-\frac{x^{\alpha}}{i+1}\right|\left|\Lambda^{\prime}\left(\frac{i+x}{i+1} a^{\varrho}+\frac{1-x}{i+1} b^{\varrho}\right)\right|^{q} \mathrm{~d} x\right)^{1 / q}\right] \\
\leq & \frac{1}{\varrho}\left[( \int _ { 0 } ^ { 1 } | \frac { 1 } { i + 2 } - \frac { x ^ { \alpha } } { i + 1 } | \mathrm { d } x ) ^ { 1 / r } \left(\int _ { 0 } ^ { 1 } | \frac { 1 } { i + 2 } - \frac { x ^ { \alpha } } { i + 1 } | \left(\frac{1}{n} \sum_{s=1}^{n}\left(1-\left(1-\left(\frac{i+x}{i+1}\right)\right)^{s}\right)\left|\Lambda^{\prime}\left(a^{\varrho}\right)\right|^{q}+\frac{1}{n} \sum_{s=1}^{n}\left(1-\left(\frac{i+x}{i+1}\right)^{s}\right)\left|\Lambda^{\prime}\left(b^{\varrho}\right)\right|^{q}\right.\right.\right. \\
& \left.\left.-\mu\left(x^{\sigma}(1-x)+x(1-x)^{\sigma}\right)\left\|b^{\varrho}-a^{\varrho}\right\|^{\sigma} \mathrm{d} x\right)^{1 / q}\right] \\
& \left.\left.-\frac{1}{(i+2)(\sigma+1)(\sigma+2)^{\prime}}\left\|b^{\varrho}-a^{\varrho}\right\|^{\sigma}\right)^{1 / q}\right] . \\
= & \frac{1}{\varrho(i+2)^{1 / r}}\left[\left(\frac{1}{n(i+2)} \sum_{s=1}^{n} \frac{(i+1)^{s}(1+s)-1}{(i+1)^{s}(1+s)}\left|\Lambda^{\prime}\left(a^{\varrho}\right)\right|^{q}+\frac{1}{n(i+2)} \sum_{s=1}^{n} \frac{(i+1)^{s}(s-i)+i^{s+1}}{(i+1)^{s}(1+s)}\left|\Lambda^{\prime}\left(b^{\varrho}\right)\right|^{q}\right.\right.
\end{aligned}
$$


Similarly,

$$
\begin{aligned}
I_{6}= & \int_{0}^{1}\left|\frac{1}{i+2}-\frac{t^{\alpha}}{i+1}\right| t^{\varrho}-1\left|\Lambda^{\prime}\left(\frac{1-t^{\varrho}}{i+1} a^{\varrho}+\frac{i+t^{\varrho}}{i+1} b^{\varrho}\right)\right| \mathrm{d} t \\
= & \frac{1}{\varrho} \int_{0}^{1}\left|\frac{1}{i+2}-\frac{x^{\alpha}}{i+1}\right| \Lambda^{\prime}\left(\frac{1-x}{i+1} a^{\varrho}+\frac{i+x}{i+1} b^{\varrho}\right) \mid \mathrm{d} x \\
\leq & \frac{1}{\varrho}\left[\left(\int_{0}^{1}\left|\frac{1}{i+2}-\frac{x^{\alpha}}{i+1}\right| \mathrm{d} x\right)^{1 / r}\left(\int_{0}^{1}\left|\frac{1}{i+2}-\frac{x^{\alpha}}{i+1}\right|\left|\Lambda^{\prime}\left(\frac{1-x}{i+1} a^{\varrho}+\frac{i+x}{i+1} b^{\varrho}\right)\right|^{q} \mathrm{~d} x\right)^{1 / q}\right] \\
\leq & \frac{1}{\varrho}\left[( \int _ { 0 } ^ { 1 } | \frac { 1 } { i + 2 } - \frac { x ^ { \alpha } } { i + 1 } | \mathrm { d } x ) ^ { 1 / r } \left(\int _ { 0 } ^ { 1 } | \frac { 1 } { i + 2 } - \frac { x ^ { \alpha } } { i + 1 } | \left(\frac{1}{n} \sum_{s=1}^{n}\left(1-\left(1-\left(\frac{1-x}{i+1}\right)^{s}\right)\right)\left|\Lambda^{\prime}\left(a^{\varrho}\right)\right|^{q}+\frac{1}{n} \sum_{s=1}^{n}\left(1-\left(\frac{1-x}{i+1}\right)\right)^{s}\left|\Lambda^{\prime}\left(b^{\varrho}\right)\right|^{q}\right.\right.\right. \\
& \left.\left.\left.-\mu\left(x^{\sigma}(1-x)+x(1-x)^{\sigma}\right)\left\|b^{\varrho}-a^{\varrho}\right\|^{\sigma} \mathrm{d} x\right)\right)^{1 / q}\right] \\
= & \frac{1}{\varrho(i+2)^{1 / r}}\left[\left(\frac{1}{i(i+2)} \sum_{s=1}^{n} \frac{(i+1)^{s}(s-i)+i^{s+1}}{(i+1)^{s}(1+s)}\left|\Lambda^{\prime}\left(a^{\varrho}\right)\right|^{q}+\frac{1}{n(i+2)} \sum_{s=1}^{n} \frac{(i+1)^{s}(1+s)-1}{(i+1)^{s}(1+s)}\left|\Lambda^{\prime}\left(b^{\varrho}\right)\right|^{q}\right.\right. \\
& \left.\left.-\frac{2 \mu}{(i+2)(\sigma+1)(\sigma+2)}\left\|b^{\varrho}-a^{\varrho}\right\|^{\sigma}\right)^{1 / q}\right],
\end{aligned}
$$

where we have used the fact that $\left|1 / i+2-x^{\alpha} / i+1\right| \leq 1 / i+2$ for all $x \in[0,1]$. Using $I_{5}$ and $I_{6}$ in $(21)$, we get the required result.

\section{Data Availability}

No data were used to support this study.

\section{Conflicts of Interest}

The authors declare that they have no conflicts of interest.

\section{Authors' Contributions}

All authors contributed equally to the writing of this paper. All authors read and approved the final manuscript.

\section{Acknowledgments}

This work was supported by the Natural Science Foundation of China (Grant nos. 61673169, 11701176, 11626101, and 11601485).

\section{References}

[1] S. Hussain, J. Khalid, and Y. Ming Chu, "Some generalized fractional integral Simpson's type inequalities with applications," AIMS Mathematics, vol. 5, no. 6, pp. 5859-5883, 2020.
[2] M. Adil Khan, J. Pečarić, and Y.-M. Chu, "Refinements of Jensen's and McShane's inequalities with applications," AIMS Mathematics, vol. 5, no. 5, pp. 4931-4945, 2020.

[3] P. Agarwal, M. Kadakal, İ. İşcan, and Y.-M. Chu, "Better approaches for $n$-times differentiable convex functions," Mathematics, vol. 8, Article ID 950, 11 pages, 2020.

[4] M. U. Awan, N. Akhtar, A. Kashuri, M. A. Noor, and Y.-M. Chu, "2D approximately reciprocal $\rho$-convex functions and associated integral inequalities," AIMS Mathematics, vol. 5, no. 5, pp. 4662-4680, 2020.

[5] S. S. Dragomir, R. P. Agarwal, and P. Cerone, "On Simpson's inequality and applications," Journal of Inequalities and Applications, vol. 5, no. 6, pp. 533-579, 2000.

[6] M. U. Awan, S. Talib, Y.-M. Chu, M. A. Noor, and K. I. Noor, "Some new refinements of Hermite-Hadamard-type inequalities involving $\Psi_{k}$-Riemann-Liouville fractional integrals and applications," Mathematical Problems in Engineering, vol. 2020, Article ID 3051920, 10 pages, 2020.

[7] A. Iqbal, M. Adil Khan, S. Ullah, and Y.-M. Chu, "Some new Hermite-Hadamard-type inequalities associated with conformable fractional integrals and their applications," Journal of Function Spaces, vol. 2020, Article ID 9845407, 18 pages, 2020.

[8] Y. Khurshid, M. Adil Khan, M. Adil Khan, and Y.-M. Chu, "Conformable fractional integral inequalities for GG- and GA-convex functions," AIMS Mathematics, vol. 5, no. 5, pp. 5012-5030, 2020.

[9] S. Rafeeq, H. Kalsoom, S. Hussain, S. Rashid, and Y.-M. Chu, "Delay dynamic double integral inequalities on time scales with applications," Advances in Difference Equations, vol. 2020, Article ID 40, 32 pages, 2020. 
[10] S. Rashid, İ. İscan, D. Baleanu, and Y.-M. Chu, "Generation of new fractional inequalities via $n$ polynomials $s$-type convexixity with applications," Advances in Difference Equations, vol. 2020, Article ID 264, 20 pages, 2020.

[11] S. Rashid, F. Jarad, and Y.-M. Chu, "A note on reverse Minkowski inequality via generalized proportional fractional integral operator with respect to another function," Mathematical Problems in Engineering, vol. 2020, Article ID 7630260, 12 pages, 2020.

[12] S. Rashid, F. Jarad, H. Kalsoom, and Y.-M. Chu, "On PólyaSzegö and Ćebyšev type inequalities via generalized $k$-fractional integrals," Advances in Difference Equations, vol. 2020, Article ID 125, 18 pages, 2020.

[13] S. Rashid, F. Jarad, M. A. Noor, H. Kalsoom, and Y.-M. Chu, "Inequalities by means of generalized proportional fractional integral operators with respect to another function," Mathematics, vol. 7, no. 12, 18 pages, Article ID 1225, 2019.

[14] S.-S. Zhou, S. Rashid, F. Jarad, H. Kalsoom, and Y.-M. Chu, "New estimates considering the generalized proportional Hadamard fractional integral operators," Advances in Difference Equations, vol. 2020, Article ID 275, 15 pages, 2020.

[15] S. Rashid, Z. Hammouch, F. Jarad, and Y.-M. Chu, "New estimates of integral inequalities via generalized proportional fractional integral operator with respect to another function," Fractals, vol. 28, no. 8, Article ID 2040027, 12 pages, 2020.

[16] S. Rashid, Z. Hammouch, D. Baleanu, and Y.-M. Chu, "New generalizations in the sense of the weighted non-singular fractional integral operator," Fractals, vol. 28, no. 7, Article ID 2040003, 11 pages, 2020.

[17] T. Abdeljawad, S. Rashid, H. Khan, and Y.-M. Chu, "On new fractional integral inequalities for $p$-convexity within intervalvalued functions," Advances in Difference Equations, vol. 2020, Article ID 330, 17 pages, 2020.

[18] L. Xu, Y.-M. Chu, S. Rashid, A. A. El-Deeb, and K. S. Nisar, "On new unified bounds for a family of functions with fractional q-calculus theory," Journal of Function Spaces, vol. 2020, Article ID 4984612, 9 pages, 2020.

[19] S. Rashid, A. Khalid, G. Rahman, K. S. Nisar, and Y.-M. Chu, "On new modifications governed by quantum Hahn's integral operator pertaining to fractional calculus," Journal of Function Spaces, vol. 2020, Article ID 8262860, 12 pages, 2020.

[20] J.-M. Shen, S. Rashid, M. A. Noor, R. Ashraf, and Y.-M. Chu, "Certain novel estimates within fractional calculus theory on time scales," AIMS Mathematics, vol. 5, no. 6, pp. 6073-6086, 2020.

[21] U. N. Katugampola, "New approach to a generalized fractional integral," Applied Mathematics and Computation, vol. 218, no. 3, pp. 860-865, 2011.

[22] M. Z. Sarikaya, E. Set, H. Yaldiz, and N. Basak, "HermiteHadamard's inequalities for fractional integrals and related fractional inequalities," Mathematical and Computer Modelling, vol. 57, no. 9-10, pp. 2403-2407, 2013.

[23] G. Hu, H. Lei, and T. S. Du, "Some parameterized integral inequalities for $p$-convex mappings via the right Katugampola fractional integrals," AIMS Mathematics, vol. 5, no. 2, pp. 1425-1445, 2020.

[24] J. Nie, J. Liu, J. F. Zhang, and T. S. Du, "Estimation-type results on the $k$-fractional Simpson-type integral inequalities and applications," Journal of Taibah University for Science, vol. 13, no. 1, pp. 932-940, 2019.

[25] C. Peng, C. Zhou, and T. S. Du, “Riemann-Liouville fractional Simpson's inequalities through generalized " $\left(m, h_{1}, h_{2}\right)$-preinvexity," Italian Journal of Pure and Applied Mathematics, vol. 38, pp. 345-367, 2017.
[26] E. Set, "New inequalities of Ostrowski type for mappings whose derivatives are $s$-convex in the second sense via fractional integrals," Computers \& Mathematics with Applications, vol. 63, no. 7, pp. 1147-1154, 2012.

[27] S.-H. Wu, M. U. Awan, M. V. Mihai, M. A. Noor, and S. Talib, "Estimates of upper bound for a $k$ th order differentiable functions involving Riemann-Liouville integrals via higher order strongly $h$-preinvex functions," Journal of Inequalities and Applications, vol. 2019, Article ID 227, 20 pages, 2019.

[28] S. Kermausuor, "Simpson's type inequalities via the Katugampola fractional integrals for $s$-convex functions," $\mathrm{Kra}$ gujevac Journal of Mathematics, vol. 45, no. 5, pp. 709-720, 2021.

[29] T. Toplu, M. Kadakal, and I. Iscan, "On n-polynomial convexity and some related inequalities," AIMS Mathematics, vol. 5, no. 2, pp. 1304-1318, 2020.

[30] M. Adil Khan, A. Iqbal, M. Suleman, and Y.-M. Chu, "Hermite-Hadamard type inequalities for fractional integrals via Green's function," Journal of Inequalities and Applications, vol. 2018, Article ID 161, 15 pages, 2018.

[31] M. Adil Khan, N. Mohammad, E. R. Nwaeze, and Y.-M. Chu, "Quantum Hermite-Hadamard inequality by means of a Green function,” Advances in Difference Equations, vol. 2020, Article ID 99, 20 pages, 2020.

[32] M. U. Awan, N. Akhtar, S. Iftikhar, M. A. Noor, and Y.-M. Chu, "New Hermite-Hadamard type inequalities for n-polynomial harmonically convex functions," Journal of Inequalities and Applications, vol. 2020, Article ID 125, 12 pages, 2020.

[33] Y. Khurshid, M. Adil Khan, and Y.-M. Chu, "Conformable integral version of Hermite-Hadamard-Fejér inequalities via $\eta$-convex functions," AIMS Mathematics, vol. 5, no. 5, pp. 5106-5120, 2020.

[34] M. A. Latif, S. Rashid, S. S. Dragomir, and Y.-M. Chu, "Hermite-Hadamard type inequalities for co-ordinated convex and qausi-convex functions and their applications," Journal of Inequalities and Applications, vol. 2019, Article ID 317, 33 pages, 2019.

[35] S. Rashid, M. A. Noor, K. I. Noor, F. Safdar, and Y.-M. Chu, "Hermite-Hadamrad type inequalities for the class of convex functions on time scale," Mathematics, vol. 7, no. 10, Article ID 956, 20 pages, 2019.

[36] H.-X. Qi, M. Yussouf, S. Mehmood, Y.-M. Chu, and G. Farid, "Fractional integral versions of Hermite-Hadamard type inequality for generalized exponentially convexity," AIMS Mathematics, vol. 5, no. 6, pp. 630-6042, 2020.

[37] G. H. Lin and M. Fukushima, "Some exact penalty results for nonlinear programs and mathematical programs with equilibrium constraints," The Journal of Optimization Theory and Applications, vol. 118, no. 1, pp. 67-80, 2003.

[38] B. T. Poljak, "Existence theorems and convergence of minimizing sequences for extremal problems with constraints," Doklady Akademii Nauk SSSR, vol. 166, pp. 287-290, 1966.

[39] I. Abbas Baloch and Y.-M. Chu, "Petrović-type inequalities for harmonic $h$-convex functions," Journal of Function Spaces, vol. 2020, Article ID 3075390, 7 pages, 2020.

[40] M. Adil Khan, M. Hanif, Z. A. Khan, K. Ahmad, and Y.-M. Chu, "Association of Jensen's inequality for $s$-convex function with Csiszár divergence," Journal of Inequalities and Applications, vol. 2019, Article ID 162, 14 pages, 2019.

[41] S. Rashid, R. Ashraf, M. A. Noor, K. I. Noor, and Y.-M. Chu, "New weighted generalizations for differentiable exponentially convex mapping with application," AIMS Mathematics, vol. 5, no. 4, pp. 3525-3546, 2020. 
[42] M.-K. Wang, H.-H. Chu, Y.-M. Li, and Y.-M. Chu, "Answers to three conjectures on convexity of three functions involving complete elliptic integrals of the first kind," Applicable Analysis and Discrete Mathematics, vol. 14, pp. 255-271, 2020.

[43] S. Zaheer Ullah, M. Adil Khan, and Y.-M. Chu, "A note on generalized convex functions," Journal of Inequalities and Applications, vol. 2019, Article ID 291, 10 pages, 2019.

[44] T.-H. Zhao, L. Shi, and Y.-M. Chu, "Convexity and concavity of the modified Bessel functions of the first kind with respect to Hölder means," Revista de la Real Academia de Ciencias Exactas, Físicas y Naturales. Serie A. Matemáticas, vol. 114, no. 2, 14 pages, Article ID 96, 2020.

[45] H. Kalsoom, M. Idrees, D. Baleanu, and Y.-M. Chu, "New estimates of $q_{1} q_{2}$-Ostrowski-type inequalities with a class of n-polynomial prevexity of function," Journal of Function Spaces, vol. 2020, Article ID 3720798, 13 pages, 2020. 